\title{
A Remark to Harris's Theorem on Percolation
}

\author{
M. Miyamoto \\ Yoshida College, Kyoto University, Kyoto, Japan \\ Received March 25, 1975
}

\begin{abstract}
Harris's theorem on percolation is generalized to a dependent case by his own method.
\end{abstract}
Let $T$ be the set of bonds in the plane square lattice $Z^{2}$. We adopt the following

\section{Notations.}

$\Omega=\{0,1\}^{T}$; the set of configurations of 0 and 1 in $T$.

$X_{t}(\omega)=\omega(t)$ for $t \in T$ and $\omega \in \Omega$.

$X=\left\{X_{t} ; t \in T\right\}$.

$\boldsymbol{X}^{-1}(i) \equiv \boldsymbol{X}^{-1}(i, \omega)=\left\{t \in T ; X_{t}(\omega)=i\right\}$ for $i=0,1$.

$P$ is a probability measure on $\Omega$.

Harris [3] proved following

Theorem. If a random field $(\Omega, P ; \boldsymbol{X})$ is independent at each $t \in T$, and if $P\left(X_{t}=0\right)=P\left(X_{t}=1\right)=1 / 2$ for every $t$, then neither $\boldsymbol{X}^{-1}(0)$ nor $\boldsymbol{X}^{-1}(1)$ has infinite connected components a.s.

His method can be applied to generalize the above to a dependent case. For $V \subset T$, let $\mathscr{B}_{V}$ be the $\sigma$-algebra generated by $\left\{X_{t} ; t \in V\right\}$, and let $\mathscr{B}_{\infty}=\bigcap_{V} \mathscr{B}_{V} c$, where $V$ runs over the set of all finite subsets of $T$. Let $\partial V$ be the set of bonds which meet bonds in $V$ at right angles.

Theorem. We assume that a random field $(\Omega, P ; \boldsymbol{X})$ satisfies the following conditions;

(1) (Spatial symmetry) $P$ is invariant under shift, rotation by right angles and reflection in the axis in $T$.

(2) (Symmetry of configurations) $P$ is invariant under interchange of 0 and 1.

(3) $P$ is everywhere dense.

(4) $\mathscr{B}_{\infty}$ is trivial, if it is measured by $P$.

(5) (The FKG inequality) If $f(\omega)$ and $g(\omega)$ are non-decreasing functions of $\omega \in \Omega$, then

$\int_{\Omega} f(\omega) g(\omega) P(d \omega) \geqq \int_{\Omega} f(\omega) P(d \omega) \cdot \int_{\Omega} g(\omega) P(d \omega)$.

(6) (Markovian property) For each $A \in \mathscr{B}_{V}$,

$$
P\left(A \mid \mathscr{B}_{V c}\right)=P\left(A \mid \mathscr{B}_{\text {oV }}\right) \text {. }
$$

Then, neither $\boldsymbol{X}^{-1}(0)$ nor $\boldsymbol{X}^{-1}(1)$ has infinite connected components a.s. 
These conditions are satisfied by the Ising model with the nearest neighbour attraction at high temperature for a suitable value of the chemical potential. (For details, see [1,2] and [4].)

Lemma 1. (Cf. Lemmata 5.1, 2, and 3 in [3].) Let $R_{1}$ be the probability that the origin $(0,0)$ belongs to an infinite connected component of $\boldsymbol{X}^{-1}(1) \cap\{x \geqq 0, y \geqq 0\}$. Then, $R_{1}=0$.

Proof. Suppose $R_{1}>0$. For a positive integer $j$, let $E_{j}$ be the event that a point $(0, j)$ belongs to an infinite connected component of $\boldsymbol{X}^{-1}(1) \cap\{x \geqq 0, y \leqq j\}$. By spatial symmetry (1), we have $P\left(E_{j}\right)=R_{1}>0$. The point-wise ergodic theorem of Birkhoff combined with (4) assures that frequency of occurence of events $E_{1}, E_{2}, \ldots$ is equal to $R_{1}$ a.s. Therefore, infinitely many $E_{j}$ 's occur a.s.

Assume that the event $E_{j}$ occurs. For a positive integer $k$, let $B_{k}$ be the event that $X_{t}=0$ for each bond $t$ in the set $\{k \leqq x \leqq k+1,0 \leqq y \leqq j\}$. Since $P\left(B_{k}\right)>0$ by (3), Birkhoff's ergodic theorem again implies that infinitely many $B_{k}$ 's occur a.s. A connected component of $\boldsymbol{X}^{-1}(1) \cap\{x \geqq 0,0 \leqq y \leqq j\}$ containing $(0, j)$ is blocked by barricades $B_{k}$, so that the infinite connected component appearing in the event $E_{j}$ crosses the $x$-axis. a.s. Thus we a.s. have infinitely many chains of 1-bonds which connect points on the $x$-axis to points on the $y$-axis.

Denote by $T^{*}$ the lattice dual to $T$. Let $X_{*^{*}}^{*}(\omega)=X_{t}(\omega)$ for a bond $t^{*}$ in $T^{*}$ which crosses a bond $t$ in $T$. A random field $X^{*}=\left\{X_{t^{*}}^{*}, t^{*} \in T^{*}\right\}$ is homomorphic to $X$.

Therefore, we a.s. have infinitely many chains of bonds in $X^{*-1}(1) \cap\left\{x \geqq-\frac{1}{2}\right.$, $\left.y \geqq-\frac{1}{2}\right\}$ which connect points on an axis $\left\{y=-\frac{1}{2}\right\}$ to points on an axis $\left\{x=-\frac{1}{2}\right\}$. By symmetry of configurations (2), we a.s. have infinitely many chains of bonds in $X^{*-1}(0) \cap\left\{x \geqq-\frac{1}{2}, y \geqq-\frac{1}{2}\right\}$ with the same property as above. Since chains in $\boldsymbol{X}^{-1}(1)$ and those in $\boldsymbol{X}^{*-1}(0)$ can not intersect with each other, there exist chains in $X^{*-1}(0) \cap\left\{x \geqq-\frac{1}{2}, y \geqq-\frac{1}{2}\right\}$ which connect points on $\left\{y=-\frac{1}{2}\right\}$ to points on $\left\{x=-\frac{1}{2}\right\}$ and do not pass a point $\left(-\frac{1}{2},-\frac{1}{2}\right)$.

The infinite connected component of $\boldsymbol{X}^{-1}(1) \cap\{x \geqq 0, y \geqq 0\}$ containing the origin necessarily crosses the above mentioned chains of bonds in $X^{*-1}(0) \cap\left\{x \geqq-\frac{1}{2}\right.$, $\left.y \geqq-\frac{1}{2}\right\}$, which is absurd. Thus, we have $R_{1}=0$.

Lemma 2. (Cf. Lemma 6.1 in [3].) Let $R_{2}$ be the probability that the origin belongs to an infinite connected component of $\boldsymbol{X}^{-1}(1) \cap\{x \geqq 0\}$. Then, $R_{2}=0$.

Proof. Suppose $R_{2}>0$. For a positive integer $j$, let $W_{j}$ be the event that a point $(j, 0)$ belongs to an infinite connected component of $\boldsymbol{X}^{-1}(1) \cap\{y \geqq 0\}$, a point $(j,-1)$ belongs to an infinite connected component of $X^{-1}(1) \cap\{y \leqq-1\}$ and $X_{t_{j}}=1$ where $t_{j}$ is a bond connecting $(j, 0)$ to $(j,-1)$. By the FKG inequality and symmetry (1) and (2), we have

$$
P\left(W_{j}\right) \geqq R_{2}^{2} \cdot P\left(X_{t_{j}}=1\right)=R_{2}^{2} / 2>0 .
$$

Therefore, Birkhoff's ergodic theorem implies that infinitely many $W_{j}$ 's occur a.s.

Assume that the event $W_{j}$ occurs. The infinite connected components of 1-bonds which appears in $W_{j}$ necessarily cross the $y$-axis both above and strictly below the origin a.s. by Lemma 1. We a.s. have chains of bonds in $\boldsymbol{X}^{-1}(1) \cap\{x \geqq 0\}$ connecting a point on the $y$-axis above the origin to a point on the $y$-axis strictly 
below the origin. Therefore by homomorphism between $\boldsymbol{X}$ and $\boldsymbol{X}^{*}$ and by symmetry (2), we have chains of bonds in $X^{*-1}(0) \cap\left\{x \geqq-\frac{1}{2}\right\}$ connecting a point on $\left\{x=-\frac{1}{2}\right\}$ above the $x$-axis to a point on $\left\{x=-\frac{1}{2}\right\}$ strictly below the $x$-axis.

The infinite connected component of $\boldsymbol{X}^{-1}(1) \cap\{x \geqq 0\}$ containing the origin necessarily crosses the above chains of bonds in $X^{*-1}(0) \cap\left\{x \geqq-\frac{1}{2}\right\}$, which is absurd. Thus, we have $R_{2}=0$.

A set $V=\{|x| \leqq n,|y| \leqq n\}$ is called a box. The integer $n$ in the definition of $V$ is denoted by $|V|$. A chain of bonds which starts at a point on the $y$-axis, ends at a point on the $y$-axis strictly below the starting point and no other bond of which crosses the $y$-axis is called a half-circuit.

Lemma 3. (Cf. Lemma 7.1 in [3].) For any box $V$, there exists a box $V^{\prime}$ such that with probability $>1 / 2$ there exists a half-circuit lying in $X^{-1}(1) \cap\{x \geqq 0\} \cap\left(V^{\prime} \backslash V\right)$, starting above the box $V$ and ending below it.

Proof. A connected component of $\boldsymbol{X}^{*-1}(0) \cap\left\{x \geqq \frac{1}{2}\right\}$ containing $(1 / 2, j+1 / 2)$ is finite a.s. by Lemma 2 , so that the point $(1 / 2, j+1 / 2)$ is surrounded a.s. by halfcircuits lying in $\boldsymbol{X}^{-1}(1) \cap\{x \geqq 0\}$. The union of all the half-circuits in $\boldsymbol{X}^{-1}(1) \cap$ $\{x \geqq 0\}$ surrounding $(1 / 2, j+1 / 2)$ is divided into connected components $C_{1}^{j}, C_{2}^{j}, \ldots$, each of which is finite a.s. by Lemma 2 . The event that there exist infinitely many components $C_{1}^{j}, C_{2}^{j}, \ldots$ belongs to $\mathscr{B}_{\infty}$, whose probability is 0 or 1 by (4).

Suppose it is 0 , i.e., there exist only finitely many components a.s. Then there exists a maximal component $C_{\infty}^{j}$. For any $j$ and $j^{\prime}$, we have $C_{\infty}^{j}=C_{\infty}^{j^{\prime}}$ or $C_{\infty}^{j} \cap C_{\infty}^{j^{\prime}}=\phi$. Along the boundary of $\bigcup_{j=-\infty}^{\infty} C_{\infty}^{j}$, there extends an infinite chain of bonds in $X^{*-1}(0) \cap\left\{x \geqq-\frac{1}{2}\right\}$, which is impossible by Lemma 2 . Therefore, there a.s. exist infinitely many components $C_{1}^{0}, C_{2}^{0}, \ldots$ surrounding the origin, all except finitely many of which are outside of the box $V$. Taking a box $V^{\prime}$ large enough, we get the result.

Let $R$ be the probability that the origin belongs to an infinite connected component of $\boldsymbol{X}^{-1}(1)$.

Lemma 4. (Cf. Lemma 7.2 in [3].) Suppose $R>0$. For any box $V$ and sufficiently large $i$, there exists a box $V^{\prime \prime}$ with $\left|V^{\prime \prime}\right|>i>|V|$ such that with probability $>R^{2} / 2^{5}$ there exists a chain in $\boldsymbol{X}^{-1}(1) \cap\left(V^{\prime \prime} \backslash V\right)$ connecting $(0, i)$ to $(0,-i)$.

Proof. Let $A$ be the event that $X_{t}=0$ for all $t \in V$, let $H_{i}$ be the event that $(0, i)$ belongs to an infinite connected component of $X^{-1}(1)$ and let $C_{i}$ be the event that $(0, i)$ belongs to an infinite connected component of $\boldsymbol{X}^{-1}(1) \cap V^{c}$. Noting that $A$ is decreasing, i.e., $\omega \leqq \omega^{\prime} \in A$ implies $\omega \in A$, we have by (5)

$$
P\left(H_{i} \cap A\right)=P\left(C_{i} \cap A\right) \leqq P\left(C_{i}\right) P(A),
$$

i.e. $P\left(H_{i} \mid A\right) \leqq P\left(C_{i}\right)$. The left-hand side of the inequality converges to $P\left(H_{0}\right)=R$, as $i \rightarrow \infty$. For sufficiently large $i$, we have $R / 2 \leqq P\left(C_{i}\right)$.

Take any box $V^{\prime}$ such that $\left|V^{\prime}\right|>i$. By Lemma 3, there exists a box $V^{\prime \prime}$ such that with probability $>1 / 2$ there exists a half-circuit lying in $\boldsymbol{X}^{-1}(1) \cap\{x \geqq 0\} \cap$ $\left(V^{\prime \prime} \backslash V^{\prime}\right)$, starting above $V^{\prime}$ and ending below $V^{\prime}$. Let $C=C(\omega)$ be the maximal one of those half-circuits.

Let $S$ be a half-circuit in $\{x \geqq 0\} \cap\left(V^{\prime \prime} \backslash V^{\prime}\right)$ from above to below $V^{\prime}$, let $S_{1}$ be the union of $S$ and its reflection in the $y$-axis and let $S_{1}^{\text {int }}$ be the interior of the 
circuit $S_{1}$. Let $D(i, S)$ be the event that there exists a chain of bonds in $\boldsymbol{X}^{-1}(1) \cap$ $\left(S_{1}^{\text {int }} \backslash V\right)$ connecting $(0, i)$ to a bond in $S$. From symmetry (1), we have, $P(D(i, S)) \geqq$ $P\left(C_{i}\right) / 2 \geqq R / 2^{2}$ and $P(D(-i, S)) \geqq R / 2^{2}$.

Let $E_{S}$ be the event that $S$ is the maximal half-circuit $C(\omega)$, and let $E_{S}^{\prime}$ be the event that $X_{t}=1$ for all $t$ on the circuit $S_{1}$ and outside of $S_{1}$. Then, we have

$$
\begin{aligned}
& P\left(D(i, S) \cap D(-i, S) \mid E_{S}\right)=P\left(D(i, S) \cap D(-i, S) \mid E_{S}^{\prime}\right) \\
& \geqq P(D(i, S) \cap D(-i, S)) \geqq P(D(i, S)) \cdot P(D(-i, S)) \geqq R^{2} / 2^{4} .
\end{aligned}
$$

The equality above is valid by the Markovian property (6) and the first two inequalities are those of FKG. Thus, we have

$$
\left.P(D(i, S) \cap D(-i, S)) \cap E_{S}\right) \geqq P\left(E_{S}\right) \cdot R^{2} / 2^{4},
$$

from which follows the desired result, since $\sum_{S} P\left(E_{S}\right)>1 / 2$.

Corollary 1. In Lemma 4 , with probability $>R^{2} / 2^{6}$ we can take the chain clockwise.

Proof is obvious from spatial symmetry (1).

Corollary 2. (Cf. Lemma 8.1 in [3].) Let $F_{V^{\prime \prime}, V}$ be the event that there exists a circuit in $\boldsymbol{X}^{-1}(1) \cap\left(V^{\prime \prime} \backslash V\right)$ surrounding the origin. For any box $V$, there exists a box $V^{\prime \prime}$ such that $P\left(F_{V^{\prime \prime}, V}\right) \geqq R^{4} / 2^{12}$.

Proof. By Corollary 1, spatial symmetry (1) and the FKG inequality (5), for a box $V$ and sufficiently large $i$, there exists a box $V^{\prime \prime}$ with $\left|V^{\prime \prime}\right|>i>|V|$ such that with probability $>\left(R^{2} / 2^{6}\right)^{2}$ there exist both clockwise chains and counterclockwise ones in $\boldsymbol{X}^{-1}(1) \cap\left(V^{\prime \prime} \backslash V\right)$ connecting $(0, i)$ to $(0,-i)$.

Lemma 5. Suppose $R>0$. Then, there exists a circuit in $X^{-1}(1)$ around the origin a.s.

Proof. Let $G$ be the event above. By Corollary 2 to Lemma 4, there exists a sequence $V_{1} \subset V_{2} \subset \ldots$ of boxes such that $P\left(F_{V_{k+1}, V_{k}}\right) \geqq R^{4} / 2^{12}$. Let $F_{k}=F_{V_{k+1}, V_{k}}$. Take arbitrary $\varepsilon>0$. By (4), there exists a sub-sequence $k_{1}<k_{2}<\ldots$ such that

$$
\left|P\left(F_{k_{n}}^{c} \cap F_{k_{n-1}}^{c} \cap \ldots \cap F_{k_{1}}^{c}\right)-P\left(F_{k_{n}}^{c}\right) P\left(F_{k_{n-1}}^{c} \cap \ldots \cap F_{k_{1}}^{c}\right)\right|<\varepsilon .
$$

Noting that $G^{c} \subset \bigcap_{n=1}^{N} F_{k_{n}}^{c}$ for arbitrary $N$, we have

$$
\begin{aligned}
P\left(G^{c}\right) & \leqq P\left(\bigcap_{n=1}^{N} F_{k_{n}}^{c}\right) \\
& \leqq P\left(F_{k_{N}}^{c}\right) P\left(\bigcap_{n=1}^{N-1} F_{k_{n}}^{c}\right)+\left|P\left(F_{k_{N}}^{c}\right) P\left(\bigcap_{n=1}^{N-1} F_{k_{n}}^{c}\right)-P\left(\bigcap_{n=1}^{N} F_{R_{n}}^{c}\right)\right| \\
& \leqq\left(1-R^{4} / 2^{12}\right) P\left(\bigcap_{n=1}^{N-1} F_{k_{n}}^{c}\right)+\varepsilon .
\end{aligned}
$$

Repeating this procedure, we have

$$
P\left(G^{c}\right) \leqq\left(1-R^{4} / 2^{12}\right)^{N-1}+\varepsilon /\left(R^{4} / 2^{12}\right) .
$$

Letting $N \rightarrow \infty$ and $\varepsilon \rightarrow 0$, we have $P\left(G^{c}\right)=0$.

Proof of Theorem. Suppose $R>0$. Then, by homomorphism between $\boldsymbol{X}$ and $\boldsymbol{X}^{*}$ and symmetry of configurations (2), there exists a circuit of 0-bonds in $X^{*}$ around $\left(-\frac{1}{2},-\frac{1}{2}\right)$ a.s.

An infinite connected component of $\boldsymbol{X}^{-1}(1)$ containing the origin crosses the above circuit in $X^{*}$, which is impossible. 


\section{References}

1. Dobrushin, R. L. : Funkt. anal. pril. 2, 44-57 (1968)

2. Fortuin, C. M., Kasteleyn, P. W., Ginibre, J.: Commun. math. Phys. 22, 89-103 (1971)

3. Harris, T.E. : Proc. Cambridge Phil. Soc. 56, 13-20 (1960)

4. Lanford III, O.E., Ruelle, D. : Commun. math. Phys. 13, $194-215$ (1968)

Communicated by G. Gallavotti

Note Added in Proof. Prof. G. Gallavotti kindly informed me that six conditions in our Theorem characterize the Ising model which has the nearest neighbour attraction, the zero external field and $T \geqq T_{c}$. 
